\title{
Spin-glass transition in porous spheres $\mathrm{BiFeO}_{3}$
}

\author{
A. V. Dmitriev ${ }^{1}$, E. V. Vladimirova ${ }^{1}$, M. A. Semkin ${ }^{2}$, A. V. Korolev ${ }^{3}$ \\ ${ }^{1}$ Institute of Solid State Chemistry, UB RAS, 91, Pervomaiskaya Str., Ekaterinburg, 620990, Russia \\ ${ }^{2}$ Ural Federal University, Department of Magnetism, 48, Kuibyshev Str., Ekaterinburg, Russia \\ ${ }^{3}$ M. N. Mikheev Institute of Metal Physics UB RAS, 18, S. Kovalevskaya Str., Ekaterinburg, 620108, Russia \\ *dmitriev@ihim.uran.ru
}

DOI 10.17586/2220-8054-2020-11-5-565-571

\begin{abstract}
Magnetic properties of porous spheres $\mathrm{BiFeO}_{3}$ have been studied at temperatures ranging from 2 to $300 \mathrm{~K}$. A transition to cluster spin glass state has been detected in the region of about $100 \mathrm{~K}$. The presence of the transition is confirmed by nonlinear variation of coercive force and the appearance of exchange displacement of magnetic hysteresis loops at temperature below $100 \mathrm{~K}$. Temperature dependence of magnetization for zero-field cooled regime exhibit a maximum at some temperature $T_{m}$. The function $T_{m}(H)$ ( $H$ is magneic field) changes in accordance with Almeida-Thouless line. The performed measurements of the frequency dependence of AC susceptibility confirm the behavior of spin glass with spin freezing temperature $T_{f}=116 \mathrm{~K}$. The critical index $z \nu=2.5$ agrees well with the mean-field theory $z \nu=2.0$.
\end{abstract}

Keywords: bismuth ferrite, magnetic properties, porous spheres, Almeida-Thouless line, spin cluster glass.

Received: 19 August 2020

Revised: 14 September 2020

\section{Introduction}

It is known that the bismuth ferrite $\mathrm{BiFeO}_{3}(\mathrm{BFO})$ is one of few materials exhibiting multiferroic properties at room temperature. However, bulk BFO is not suitable for application because of a spin cycloid with period length of $62 \mathrm{~nm}$ in its magnetic structure [1] that reduces weak ferromagnetism to zero, thus preventing the appearance of a linear magnetoelectric effect [2]. Reduction to nanodimensionis an effective method of ferromagnetic order formation in BFO system due to an increase of the fraction of uncompensated spins and suppression of spiral order as a result of reduction of crystallite sizes [3,4]. Both in thin BFO films and nanocrystals, magnetic switching can be induced by electric field $[5,6]$, which opens up prospects for practical application of BFO in magnetic sensors, spintronics and data storage devices [1].

Recently, the study of magnetic properties was focused on BFO films and nanopowders. Different experiments, including direct magnetic measurements, suggest several magnetic transitions in the temperature range from 5 to $250 \mathrm{~K}$ [7-9]. The nature of these transitions is not entirely understood. They can be related either to spin orientation variation or to transition to spin glass state. The results of neutron diffraction studies and investigations of Raman spectra and dielectric properties revealed the presence of a phase transition connected with spin reorientation at $150 \mathrm{~K}$ [10-12]. Spin reorientation transition facilitates transition to spin glass state at further decrease of temperature [12]. The majority of researchers of nanocrystalline powders believe that magnetic properties can be explained in the model of antiferromagnetic nucleus with ferromagnetic shell. At the same time, some questions remain undetermined. Unusual effects of exchange displacement of hysteresis loop above transition temperature to spin glass state and nonmonotonic variation of coercive force require further research $[9,13]$. Park et al. [3] inferred that the anomalous magnetization behavior in BFO nanoparticles arises from a complex interplay between the finite size effects, interparticle interaction, and a random distribution of anisotropy axis in nanoparticle assemblies. The systems of interacting magnetic nanoparticles may demonstrate a large variety of phenomena, which are of interest both for fundamental scientific studies and future practical applications of magnetic nanoparticles [14].

Examples of nanoparticles assembling are such materials as nanofibers [15], nanotubes [16], hollow spheres [17, $18]$ etc. consisting of randomly oriented nanocrystals [15,18]. Earlier we showed that spherical agglomerates of about $1 \mu \mathrm{m}$ in size possess at room temperature weak ferromagnetism typical of nanoparticles making up spheres [18]. At the same time, there are no available data on the presence of low-temperature phase transitions typical of nanoparticles. At present, almost no experimental studies of low-temperature magnetic properties of nanostructured BFO are available.

In this work, the magnetic properties of spherical porous BFO agglomerates produced by ultrasonic spray pyrolysis are comprehensively studied in the temperature range $2-300 \mathrm{~K}$. Based on the results of field and temperature dependences of magnetization, magnetic AC susceptibility measurements we have established the existence of a phase transition to spin glass state in the examined materials. 


\section{Experimental}

Spherical BFO agglomerates were synthesized by ultrasonic spray pyrolysis (USP) from a solution of iron and bismuth nitrates with addition of 6 moles of tartaric acid (TA) per 1 mole of $\mathrm{BiFeO}_{3}$. The synthesis procedure is described in detail in works $[18,19]$.

X-ray powder diffraction (XRPD) analysis was carried out using an XRD-7000 (SHIMADZU) diffractometer with a secondary monochromator with $\mathrm{Cu} \mathrm{K} \alpha$ radiation in the $2 \theta$ range from 20 to $80^{\circ}$ with a step of $0.03^{\circ}$. Structural investigations were performed in transmission geometry using an automatic STOE STADI-P diffractometer equipped with a linear mini-PSD detector using $\mathrm{Cu} \mathrm{K} \alpha_{1}$ radiation in the $2 \theta$ range from 5 to $120^{\circ}$ with a step of $0.02^{\circ}$. Polycrystalline silicon $(a=5.43075(5) \AA)$ was used as an external reference.

The morphology of the samples and 3D FIB-SEM tomography study were carried out using a TESCAN LYRA 3 dual beam system.

Magnetic measurments were carried out using setups of Quantum Design - MPMS-XL-7 (UFU) as well as PPMS-9 of the Collaborative Access Center "Testing Center of Nanotechnology and Advanced Materials" of the Institute of Metal Physics.

\section{Results and discussion}

The X-ray diffraction patterns of BFO sample are presented in Fig. 1(a). All the basic reflections correspond to ferrite $\mathrm{BiFeO}_{3}$ with perovskite structure and space group R3c (ICSD Collection Code 15299). The lattice parameters are calculated by way of full-profile analysis of X-ray diffraction patterns with the use of structural models of the corresponding compounds $\left(a_{h e x}=5.58851, c_{h e x}=13.7852\right)$. The sizes of coherent scattering regions (CSR) were found by the Le Bail method using the Klug \& Alexander approach [20], allowing the contribution of size effects to FWHM to be determined. The value of CSR, calculated from the X-ray diffraction patterns, is proportional to the sizes of nanocrystallites composing the agglomerates $(C S R=26.2 \mathrm{~nm})$. Therefore, hereafter, we shall consider that the CSR value is equal to nanocrystallite size. For the analysis of lattice distortions we used the rhombohedral cell parameters $a=a_{\text {hex }} / \sqrt{2}$ and $c=c_{\text {hex }} / 2 \sqrt{3}(c / a=1.0070)$. The obtained nanocrystal sizes and the lattice distortion parameter coincide with the results of structural studies of nanopowders reported in work [21]. Earlier, we showed [18] that the samples synthesized by USP technology contain no oxygen vacancies, and all Fe ions have valence $3+$.

The electron microscopy study of the powders reveals that all the samples produced from solutions with TA concentration $6 \mathrm{~mol} \mathrm{TA} / \mathrm{mol} \mathrm{BFO}$ represent porous agglomerates of spherical shape. The mean diameter of the agglomerates determined from the results of $300-400$ measurements amounted to about 1.2 microns (Fig. 1(b)). The internal structure of agglomerates was studied by the SEM method with focused gallium ion beam polishing (FIB). Fig. 1(c) displays a SEM image of an agglomerate after cross-section with preliminary application of a platinum mask with the use of a gas injecting system (GIS). The platinum mask is a heat sink and it also protects particle from melting and mechanical failure during ion beam etching. The image of particle after polishing (Fig. 1(c)) proves that the agglomerates have a porous cellular structure throughout.

Figure 2(a) demonstrates magnetic hysteresis loops of the examined powder. Note that the loops shape and the magnetization values practically coincide with the field dependences of magnetization of BFO nanopowders of size smaller than $40 \mathrm{~nm}$ reported in work [3]. The coercive force values at room temperature are also similar. The temperature dependence $H_{C}(T)$ exhibits a minimum near $100 \mathrm{~K}$. An analogous $H_{C}(T)$ dependence is observed in nanocrystals [9] with a minimum at $50-60 \mathrm{~K}$. The authors of work [8] attribute the $H_{C}(T)$ minimum to the transition of iron ions on the surface of nanoparticles to spin cluster glass state, which is confirmed by the exchange displacement of hysteresis loops. It is interesting to study the exchange displacement in porous spheres BFO. The inset in Fig. 2(a) shows $M(H)$ loops for ZFC and FC sample $\left(H_{F C}=70 \mathrm{kOe}\right.$ ) at $2 \mathrm{~K}$ (the sample was field cooled from 300 to $2 \mathrm{~K}$ ). It can be seen that the FC hysteresis loop is displaced towards negative field and positive magnetization, whereas in $\mathrm{ZFC}$ process the loop is centered around the origin of coordinates.

Note that the process of field cooling adopted in this study is not a traditional FC, since $T_{N}$ of $\mathrm{BFO}$ is much higher than $300 \mathrm{~K}$. Thus, the exchange anisotropy established in this system without usual FC can be related to spontaneous effect of exchange displacement [7]. The dependence of $H_{E}(T)$ on cooling field of $70 \mathrm{kOe}$ is presented in Fig. 2(b). As the temperature is raised, $H_{E}$ decreases abruptly and becomes equal to zero at $T>100 \mathrm{~K}$.

If the minimum on the temperature dependence of $H_{C}(T)$ in the region of $100 \mathrm{~K}$ is related to the transition to spin glass state, then near this temperature there should be a temperature-dependent anomaly in the magnetization variation in $\mathrm{ZFC}$ mode.

Figure 3(a) demonstrates ZFC and FC magnetization curves of spherical agglomerates of BFO in 500 Oe field. As seen from Fig. 3(a), there is a divergence of ZFC and FC curves, which begins at $300 \mathrm{~K}$ and increases gradually with decreasing temperature. The observed splitting of the ZFC and FC curves at low temperatures is a distinctive feature 

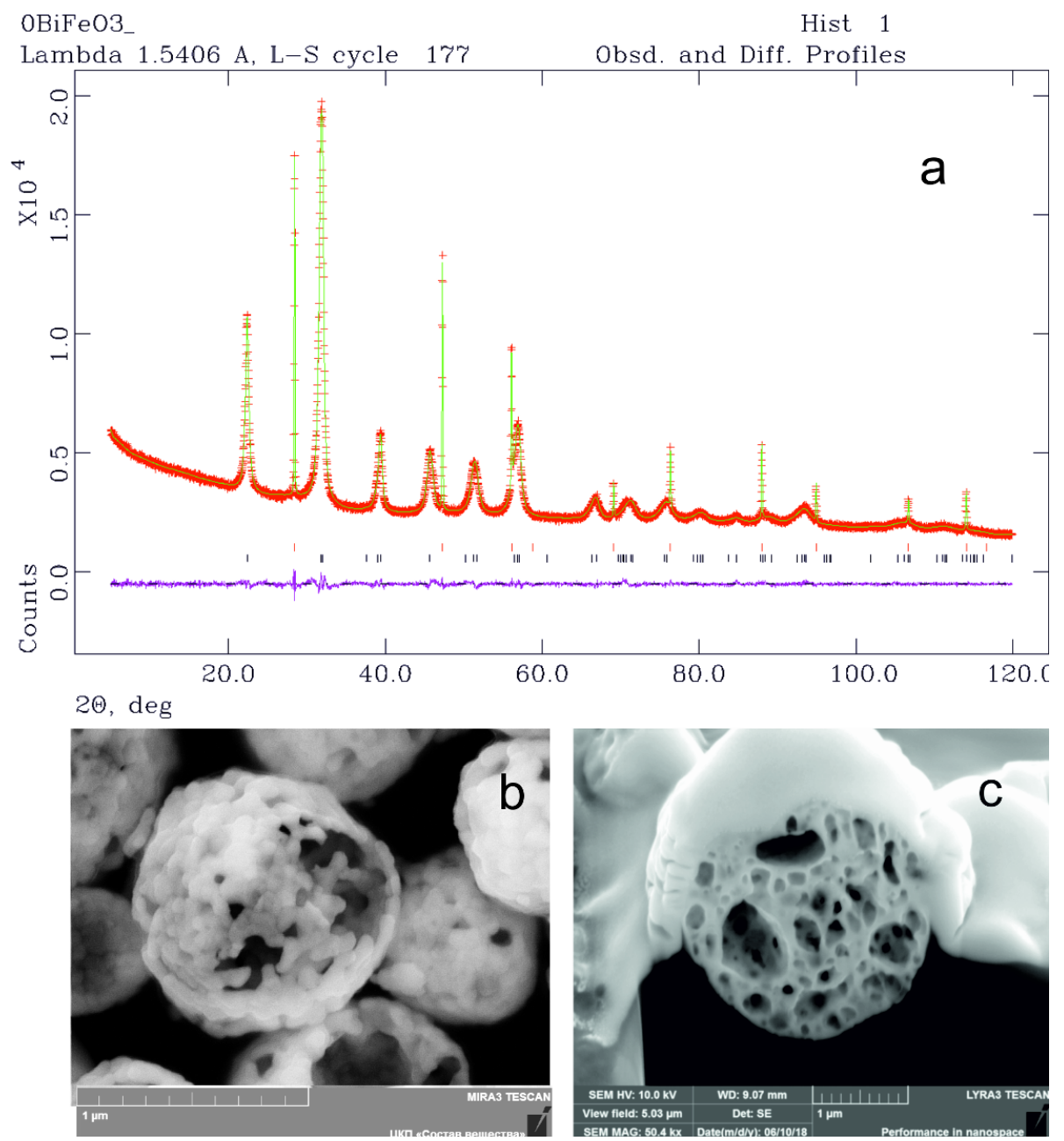

FIG. 1. a - Experimental (crosses), calculated (solid line), and difference (bottom line) XRPD patterns for $\mathrm{BiFeO}_{3}$ and $\mathrm{Si}$ used as internal standard. Series of tick marks correspond to the Bragg reflections. $\mathrm{b}$ - SEM image of BFO agglomerates. $\mathrm{c}$ - SEM image of agglomerate after gallium ion beam polishing for $15 \mathrm{~min}$. A platinum mask is applied on top

of spin glass-like state and can be due to local spin clusterization [22]. In addition, we observe a sharp inflection on the ZFC curve at about $120 \mathrm{~K}$, which can be ascribed to a typical blocking process of superparamagnetic spin moments assembly [23]. On the contrary, these moments are arranged parallel to the applied field during FC measurement, which leads to a large divergence of FC and ZFC curves below the freezing temperature. Similar changes in FC and ZFC are observed in BFO nanopowders [7-9]. A distinctive feature is that the maximum on the ZFC curve in nanoparticles is observed as a rule at a temperature of about $50 \mathrm{~K}$.

The ZFC and FC magnetization characteristics of spherical BFO agglomerates were additionally studied by applying displacement magnetic field of different strength from 0.1 to $30 \mathrm{kOe}$. The splitting temperature $T_{\text {irr }}(H)$ (irreversibility in ZFC) in all fields was observed in the temperature range $250-300 \mathrm{~K}$. We could not establish the field dependence of $T_{i r r}(H)$ because of gradual divergence (perhaps this is due to the absence of heating above $300 \mathrm{~K}$ ). The temperature dependences of ZFC magnetization in different fields are presented in Fig. 3(b).

The temperature $\left(T_{m}\right)$ of ZFC plot, $M(T)$, is displaced towards lower temperatures when the magnetic field $H$ increases. For fields smaller than $10-20 \mathrm{kOe}$ this displacement is described by Almeida-Thouless dependence (AT line):

$$
H=A\left[1-T_{m}(H) / T(0)\right]^{3},
$$

where $T_{m}(H)$ and $T(0)$ are the field dependence and the maximum temperature at $H=0$, respectively. Eq. (1) establishes the boundary of transition to superparamagnetic state or the appearance of spin glass (AT line). As seen from Fig. 3(c), the $T_{\max }(H)$ dependence can be divided into 2 parts: the first - in the interval $3-20 \mathrm{kOe}$; the second in the interval $0-3 \mathrm{kOe}$. Data fitting by AT equation yields $T_{1}(0)=97 \mathrm{~K}$ for magnetic fields $3-20 \mathrm{kOe}$ and 
a

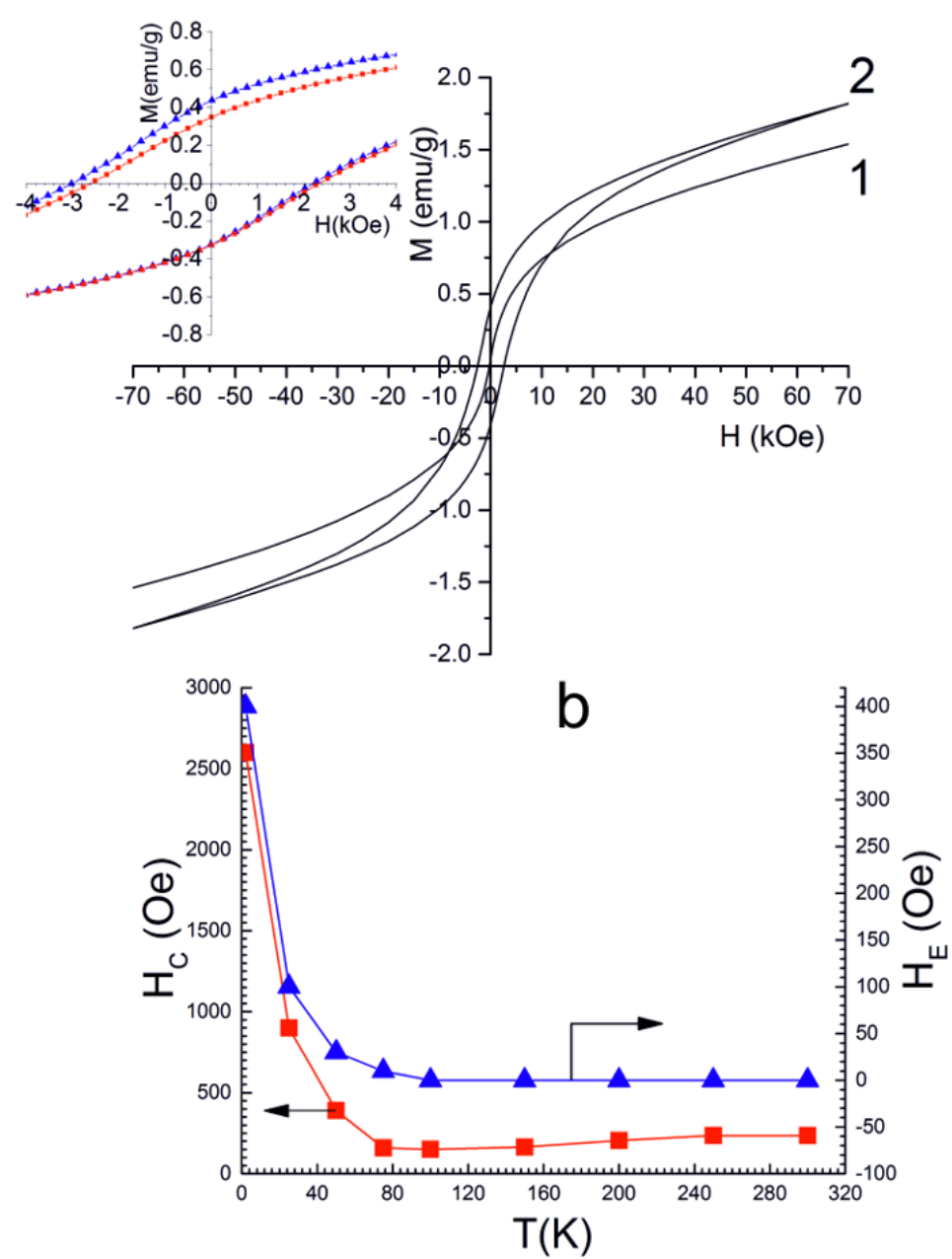

FIG. 2. a - Field dependences of magnetization. $1-300 \mathrm{~K} ; 2-2 \mathrm{~K}$. Inset: loops obtained in ZFC and FC regimes in field of $70 \mathrm{kOe} . \mathrm{b}-$ Temperature dependences of coercive force $H_{C}$ and displacement fields $H_{E}$

$T_{2}(0)=140 \mathrm{~K}$ for fields $0-3 \mathrm{kOe}$. An analogous inflection on the AT line in the region of $1-2 \mathrm{kOe}$ is observed in films and nanocrystals [8,24].

In order to find out, to which state the low-temperature phase corresponds, we measured the frequency-dependent variable of magnetic AC susceptibility $[\chi(\omega, T)]$ in zero DC field.

Figure 4(a) shows the temperature dependences of the real part $\chi^{1}$ of susceptibility to alternating field for porous spheres BFO at different frequencies at $H=$ const $\approx 0$. It is seen that the position of the $\chi^{1}(T)$ peak, corresponding to the freezing temperature $T_{f}$, is displaced towards higher temperatures with increasing frequency. The shift of the peak temperature $\chi^{1}(\omega, T)$ was analyzed in terms of the empirical parameter of frequency-dependent sensitivity $K=\Delta T_{f} /\left(T_{f} \Delta \ln \omega\right.$ ) (so-called Mydosh parameter), lying in the range $0.001-0.01$ and $0.01-0.1$ [25] for spin glass freezing and SPM blocking, respectively. In case of BFO, the $K$ parameter turns out to be equal to 0.06, which is higher than in usual spin glass system and fits better SPM blocking. From Fig. 3(a) it is possible to obtain the temperature dependences of relaxation time $\tau=1 / 2 \pi f$ (Fig. 4(b)). Since the Mydosh parameter corresponds to SPM, then in accordance with the Neel superparamagnetic model the relaxation process should follow the Arrhenius equation [26]:

$$
\tau=\tau_{0} \exp \left(E_{a} / k_{B} T_{f}\right)
$$

where $\tau_{0}$ is the relaxation time constant, $E_{a}$ is the anisotropy energy, and $k_{B}$ is the Boltzmann constant. The results of fitting with $E_{a} / k_{B}=3249 \pm 6 \mathrm{~K}$ and $\tau_{0}=2.3 \cdot 10^{-15}$ are presented in Fig. 4(b). From the obtained values it follows that BFO spheres are not Neel SPM system [27], since for superparamagnetic relaxation $\tau_{0}$ should be $10^{-10}$ $10^{-13} \mathrm{~s}$. The temperature dependence of relaxation time can be also approximated with the use of Vogel-Fulcher or 

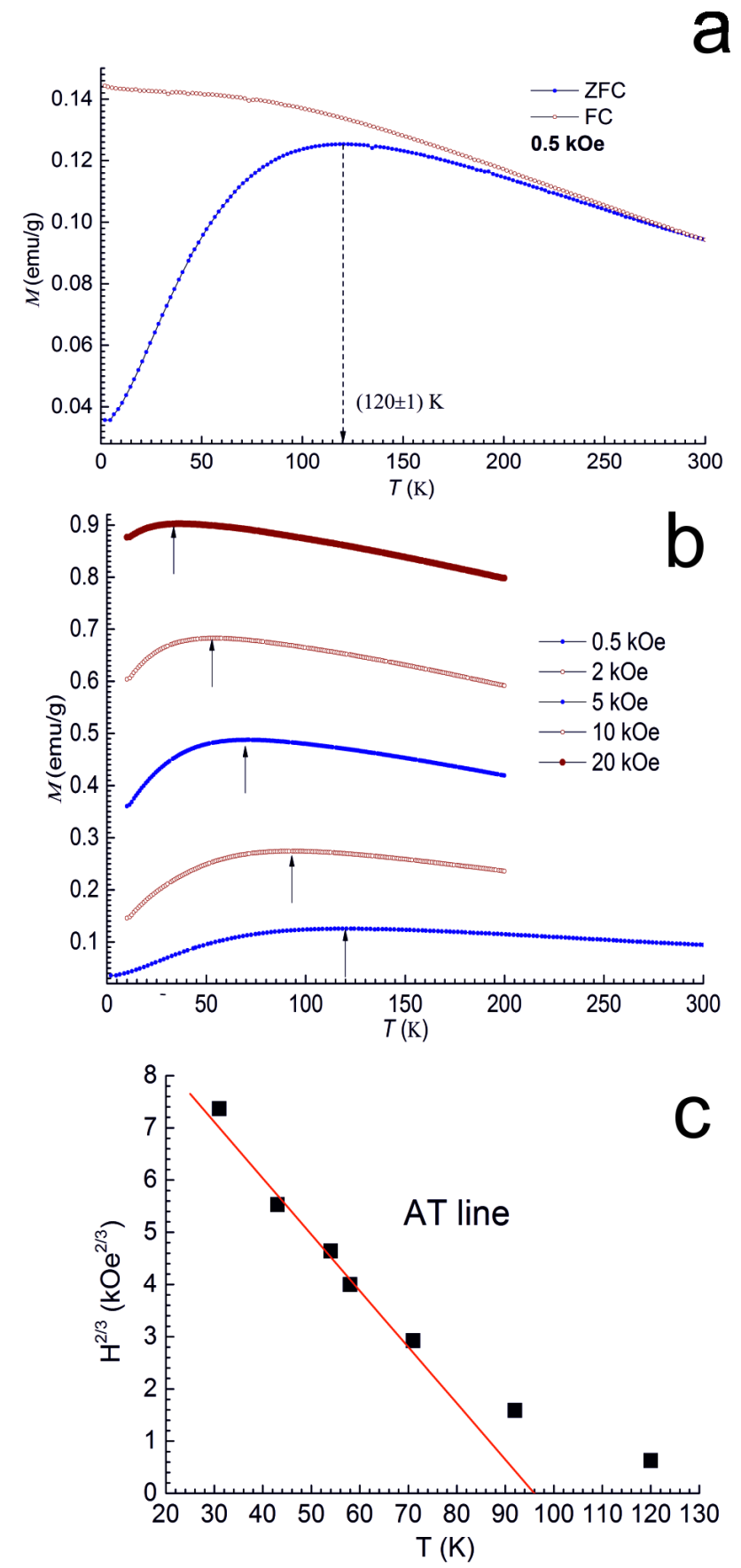

FIG. 3. Almeida-Thouless line is shown by dotted line. a - Temperature dependences of FC and ZFC magnetization at values of magnetic field strength $0.5 \mathrm{KOe}$; $\mathrm{b}$ - Temperature dependences of ZFC magnetization at different values of magnetic field strength; $c$ - Almeida-Thouless line (AT)

power laws. For the Vogel-Fulcher law [28]:

$$
\tau=\tau_{0} \exp \left[E_{a} / k_{B}\left(T_{f}-T_{V F}\right)\right],
$$

where $T_{V F}$ is the representative Vogel-Fulcher temperature. The best fit between $\tau(T)$ data and eq. (3) yields $E_{a} / k_{B}=86 \pm 6 \mathrm{~K}, T_{V F}=109 \pm 0.2 \mathrm{~K}$ and $\tau_{0}=(2.7 \pm 0.06) \times 10^{-5}$ s. For the power law [29]:

$$
\tau=\tau_{0}\left(T_{f} / T_{g}-1\right)^{z \nu},
$$

where $T_{g}$ is the glass transition temperature (static freezing temperature), $v$ is the critical index describing the correlation distance growth, and $z$ is the dynamic index describing the relaxation retardation. The power law is observed 
at $T_{g}=116 \pm 11 \mathrm{~K}, \tau_{0}=(6.7 \pm 0.13) \times 10^{-6} \mathrm{~s}$ and $z \nu=2.5 \pm 1$. The values of $\tau_{01}$ and $\tau_{02}$ for both power and VF laws fall in the typical category of cluster glass (CG) $\left(\tau=10^{-5}-10^{-10} \mathrm{~s}\right)$ for concentrated systems [30], rather than in canonical spin glasses in diluted systems. Thus, both the power law and V-F dynamics prove that the frequency-dependent anomaly in $\chi(\omega, T)$ of spherical samples BFO is related to the transition to cluster spin glass state.
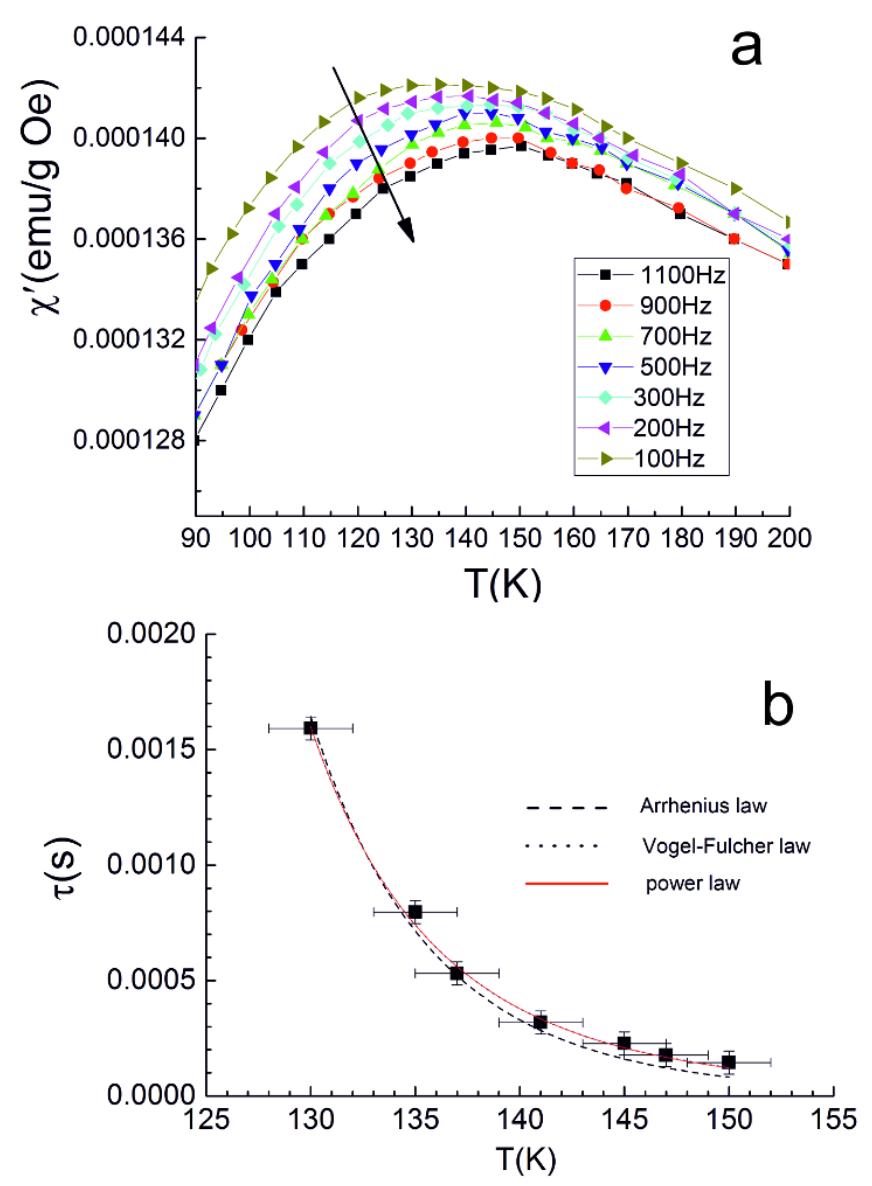

FIG. 4. a - The temperature dependence of the real part of AC magnetic susceptibility at different frequencies. The arrow shows the direction of frequency enhancement and the shift of the maximum. $\mathrm{b}$ - The temperature dependence of relaxation time

\section{Conclusion}

As a result of the research, it was established: (i) the presence of a minimum in the temperature dependence of $H_{s}(T)$ at $100 \mathrm{~K}$ and the appearance of an exchange bias increasing with decreasing temperature; (ii) FC and ZFC magnetizations differ greatly below $250-300 \mathrm{~K}$; (iii) a maximum is observed in the temperature dependence of the magnetization $\mathrm{ZFC}$ at a certain value of $T_{m}$, and with an increase in intensity $H$, the value of $T_{m}$ decreases; (iv) a maximum near $120 \mathrm{~K}$ is observed on the temperature dependence of AC magnetic susceptibility, which shifts towards low temperatures when the frequency decreases. The intensity of the maximum of susceptibility lowers if frequency increases, which is typical of glass-like state that becomes less sensitive with enhancement of frequency.

The experimental data obtained in this work prove that in the temperature region of about $100 \mathrm{~K}$ there takes place a transition to spin glass state (or at least nonergodic behavior) in porous spheres $\mathrm{BiFeO}_{3}$. The high values of relaxation time $\tau_{0}$ of the order of $10^{-6}-10^{-5} \mathrm{~s}$ allow one to draw a conclusion about the formation of cluster spin glass. The critical index describing the retardation of vitreous solid dynamics is $z \nu \approx 2.5$, which is much closer to the value obtained in the medium field system (where $z \nu \approx 2.0)$ than in the classical Ising short-range model $(z \nu \approx 7-$ $10)$.

Note the presence of a knee on the $T_{m}(H)$ dependence in the region of fields $1-2 \mathrm{kOe}$. An analogous inflection is observed in nanopowders and films. The reasons of this inflection are not clear and require further studies. 
As distinct from nanocrystals, porous spheres $\mathrm{BiFeO}_{3}$ have a higher temperature of transition to spin glass state and at temperatures above the glass transition temperature there is no exchange displacement of hysteresis loops. There is also no phase transition to the ferromagnetic state at $5 \mathrm{~K}$.

\section{Acknowledgements}

This work was supported by a research program at the Institute of Solid State Chemistry. The authors are grateful to Dr. Maria V. Lukashova (OOO “Tescan”, Saint Petersburg, Russia) for scanning microscopy and 3D FIB-SEM tomography.

\section{References}

[1] Catalan G., Scott J.F. Physics and applications of bismuth ferrite. Adv. Materials, 2009, 21, P. $2463-2485$.

[2] Pyatakov A.P., Zvezdin A.K. Magnetoelectric and multiferroic media. Uspekhi fizicheskikh nauk, 2012, 182, P. 593-620. (in Russian)

[3] Tae-Jin Park, Papaefthymiou G.C., et al. Size-dependent magnetic properties of single-crystalline multiferroic BiFeO 3 nanoparticles. Nano Lett., 2007, 7 (3), P. 766-772.

[4] Ederer C., Spaldin N.A. Weak ferromagnetism and magnetoelectric coupling in bismuth ferrite. Phys. Rev. B, $2005,71,060401$.

[5] Carranza-Celis D., Cardona-Rodríguez A., et. al. Control of Multiferroic properties in $\mathrm{BiFeO}_{3}$ nanoparticles. Scientific Reports, 2019 , 9, 3182.

[6] Sando D., Barthéléemy A., Bibes M. $\mathrm{BiFeO}_{3}$ epitaxial thin films and devices: past, present and future. J. Phys.: Condens. Matter, 2014, 26, 473201.

[7] Sining Dong, Yiping Yao, et al. Dynamic properties of spin cluster glass and the exchange bias effect in $\mathrm{BiFeO}_{3}$ nanocrystals. Nanotechnology, 2011, 22, 385701 .

[8] Manoj Singh K., Prellier W., et al. Spin-glass transition in single-crystal $\mathrm{BiFeO}_{3}$. Phys. Rev. B, 2008, 77, 144403.

[9] Fengzhen Huang, Xingyu Xu, et al. The exchange bias behavior of $\mathrm{BiFeO}_{3}$ nanoparticles with natural core-shell structure. Scientific Reports, $2018, \mathbf{8}, 2311$

[10] Cazayous M., Gallais Y., et al. Possible Observation of Cycloidal Electromagnons in $\mathrm{BiFeO}_{3}$. Phys. Rev. Lett., $2008,101,037601$.

[11] Jarrier R., Marti X., et. al. Surface phase transitions in $\mathrm{BiFeO}_{3}$ below room temperature. Phys. Rev. B, $2012, \mathbf{8 5}, 184104$.

[12] Goswami S., Bhattacharya D. Magnetic transition at $\sim 150 \mathrm{~K}$ in nanoscale $\mathrm{BiFeO}_{3}$. Journal of Alloys and Compounds, 2018,783 , P. $277-282$.

[13] Lomanova N.A., Tomkovich M.V., et al. Magnetic properties of $\mathrm{Bi}_{1-x} \mathrm{Ca}_{x} \mathrm{FeO}_{3-\delta}$ nanocrystals. J. Nanopart. Res., 2018,20 (17), P. $12-17$.

[14] Komogortsev S.V., Fel'k V.A., Li O.A. The magnetic dipole-dipole interaction effect on the magnetic hysteresis at zero temperature in nanoparticles randomly dispersed within a plane. Journal of Magnetism and Magnetic Materials, 2019, 473 (1), P. 410-415.

[15] Guilherme H.F. Melo, Joro P.F. Santos, et al. Correlation between electrospinning parameters and magnetic properties of BiFeO 3 nanofibers Electrospinning, 2017, 1, P. 73-86.

[16] Lei Wu, Wenbo Sui, et al. One-dimensional $\mathrm{BiFeO}_{3}$ Nanotubes: Preparation, Characterization, Improved Magnetic Behaviors, and Prospects. Applied Surface Science, 2016, 384, P. 368-375.

[17] Dmitriev A.V., Vladimirova A.V., et al. Hollow spheres of $\mathrm{BiFeO}_{3}$ : Synthesis and properties. Journal of Alloys and Compounds, 2018, 743, P. 654-657.

[18] Dmitriev A.V., Vladimirova A.V., et al. Synthesis of hollow spheres of $\mathrm{BiFeO}_{3}$ from nitrate solutions with tartaric acid: Morphology and magnetic properties. Journal of Alloys and Compounds, 2019, 777, P. 586-592.

[19] Dmitriev A.V., Vladimirova A.V., et al. Self-Assembly of Hollow Bismuth Ferrite Spheres from Nitrate Solutions. Journal of Electronicmaterials, 2019, 48 (8), P. 4959-4969.

[20] Klug H.P., Alexander L.E. X-ray Diffraction Procedures for Polycrystalline and Amorphous Materials. Wiley-Interscience, New York, 1974, $992 \mathrm{p}$.

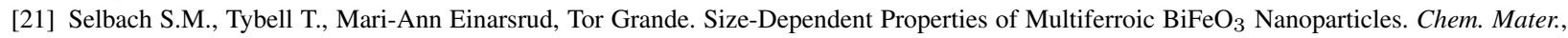
2007, 19, P. 6478-6484.

[22] Nakamura S., Soeya S., Ikeda N., Tanaka M. Spin-glass behavior in amorphous BiFeOs. J. Appl. Phys., 1993, 74, P. 5652-5657.

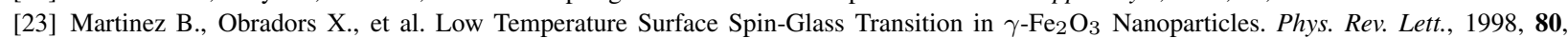
P. 181-183.

[24] Manoj K. Singh, Ram S Katiyar, Prellier W., Scott J.F. The Almeida-Thouless line in BiFeO 3 : is bismuth ferrite a mean field spin glass? J. Phys.: Condens. Matter, 2009, 21, 042202.

[25] Mydosh J.A. Disordered magnetism and spin glasses. J. Magn. Magn. Mater., 1996, 158, P. $606-610$.

[26] Bedanta S., Kleemann W. Supermagnetism. J. Phys. D: Appl. Phys., 2009, 42, 013001.

[27] Mukadam M.D., Yusuf S.M., et al. Dynamics of spin clusters in amorphous $\mathrm{Fe}_{2} \mathrm{O}_{3}$. Phys. Rev. B, $2005,72,174408$.

[28] Tholence J.L. On the frequency dependence of the transition temperature in spin glasses. Solid State Commun., 1993, 88, P. 917-921.

[29] Hohenberg P.C., Halperin B.I. Theory of Dynamic Critical Phenomena. Rev. Mod. Phys., 1977, 49, P. 435-479.

[30] Pimenov A., Mukhin A.A., et al. Possible evidence for electromagnons in multiferroicmanganites. Nat. Phys., 2006, 2, P. 97-100. 